\title{
An Intrinsic Neuronal Oscillator Underlies Dopaminergic Neuron Bursting
}

\author{
Christopher A. Deister, Mark A. Teagarden, Charles J. Wilson, and Carlos A. Paladini \\ Neurosciences Institute, The University of Texas at San Antonio, San Antonio, Texas 78249
}

Dopaminergic neurons of the ventral midbrain fire high-frequency bursts when animals are presented with unexpected rewards, or stimuli that predict reward. To identify the afferents that can initiate bursting and establish therapeutic strategies for diseases affected by altered bursting, a mechanistic understanding of bursting is essential. Our results show that bursting is initiated by a specific interaction between the voltage sensitivity of NMDA receptors and voltage-gated ion channels that results in the activation of an intrinsic, action potential-independent, high-frequency membrane potential oscillation. We further show that the NMDA receptor is uniquely suited for this because of the rapid kinetics and voltage dependence imparted to it by $\mathrm{Mg}^{2+}$ ion block and unblock. This mechanism explains the discrete nature of bursting in dopaminergic cells and demonstrates how synaptic signals may be reshaped by local intrinsic properties of a neuron before influencing action potential generation.

\section{Introduction}

Dopaminergic neurons fire bursts in vivo with intraburst frequencies that range from 14 to $30 \mathrm{~Hz}$ (Grace and Bunney, 1984; Schultz, 1986; Hollerman and Schultz, 1998; Hyland et al., 2002). Phasic burst firing is essential to reinforcement learning (Schultz, 1997; Zweifel et al., 2009), but at the cellular level it is not known how dopaminergic cells generate this complex signal. Do dopaminergic cells simply relay a signal generated elsewhere, or do they synthesize its reward-related signal from a diverse set of inputs? Insight into the burst mechanism will reveal clues about what afferents, conveying different psychological information, are necessary for bursting.

Reports show that activation or blockade of NMDA, but not AMPA, receptors (NMDAR, AMPAR) triggered or blocked burst firing, respectively, in vivo (Overton and Clark, 1992; Chergui et al., 1993; Tong et al., 1996a). Initial in vitro studies showed that tonic burst firing could be induced by bath application of NMDA (Johnson et al., 1992; Seutin et al., 1993). Later studies showed that phasic bursts like those seen in vivo could be evoked by electrical stimulation of glutamatergic afferents or iontophoresis of glutamate in slices (Morikawa et al., 2003; Blythe et al., 2007). Thus, burst firing is critically dependent on NMDAR activation, but under some conditions AMPARs may also be involved.

Bursts have been proposed to arise from a large sustained depolarization that could arise from a large-amplitude, longduration EPSP (Tong et al., 1996b), or a synaptically triggered intrinsic plateau potential (Li et al., 1996; Canavier, 1999). How-

Received Aug. 18, 2009; revised Nov. 4, 2009; accepted Nov. 9, 2009.

This work was supported by National Institute of Mental Health Grant MH079276 (C.A.P.), National Institute of Neurological Disorders and Stroke Grant NS47085 (C.J.W.), and Ruth L. Kirschstein National Research Service Award F31NS064755 (C.A.D.).

Correspondence should be addressed to Carlos A. Paladini, The University of Texas at San Antonio, Neurosciences Institute, One UTSA Circle, San Antonio, TX 78249. E-mail: carlos.paladini@utsa.edu.

DOI:10.1523/JNEUROSCI.4053-09.2009

Copyright $\odot 2009$ Society for Neuroscience $\quad 0270-6474 / 09 / 2915888-10 \$ 15.00 / 0$ ever, even large depolarizing currents increase the firing rate only slightly (Yung et al., 1991) because spiking fails before typical burst rates are obtained, a phenomenon called depolarization block (Grace and Bunney, 1986; Richards et al., 1997). This suggests that plateau potentials or long-duration EPSPs are unsuited for generating bursts.

Another mechanism proposes that dendritic NMDAR activation transiently forces the neuron to fire at the frequency of an otherwise subliminal dendritic oscillator (Kuznetsov et al., 2006). Because of the voltage dependence imparted by $\mathrm{Mg}^{2+}$ ions (Mayer et al., 1984; Nowak et al., 1984), the NMDAR contributes an inward current at depolarized potentials but is rapidly blocked by $\mathrm{Mg}^{2+}$ when hyperpolarized, allowing the neuron's intrinsic hyperpolarizing currents to minimize depolarization block. This mechanism of burst firing could explain both its transient nature and apparent affinity for NMDAR activation. However, Kuznetsov et al. (2006) assumed that the hyperpolarizing current responsible for the fast oscillation was dependent on dendritic small-conductance calcium-dependent potassium (SK) channels, but blocking SK channels enhances bursts firing in vivo (Ji and Shepard, 2006).

We show that NMDAR activation triggers burst firing, even at somatic locations. The frequency of burst firing and the oscillation that underlies it is regulated by the degree of NMDAR voltage sensitivity. Burst firing is sensitive to blockade of L-type $\mathrm{Ca}^{2+}$ channels, but is enhanced by blocking SK channels. Thus, the unique role NMDAR activation plays in burst firing is the result of the engagement of an intrinsic high-frequency membrane potential oscillation that is independent of the slow oscillation that drives spontaneous firing.

\section{Materials and Methods}

Slice preparation. Sprague Dawley rats (Charles River Laboratories) aged between 15 and $37 \mathrm{~d}$ were used in these studies. Animal handling and all procedures were approved by institutional animal care and use committees and were in accordance with National Institutes of Health guidelines. 
All efforts to minimize both the discomfort to and the number of animals were made. Animals were anesthetized with a lethal dose of ketamine and xylazine $(160 \mathrm{mg} / \mathrm{kg}$ and $24 \mathrm{mg} / \mathrm{kg})$ and then rapidly decapitated. Brains were quickly removed and placed into ice-cold artificial CSF (ACSF) consisting of the following (in mM): $126 \mathrm{NaCl}, 2.5 \mathrm{KCl}, 1.25 \mathrm{NaH}_{2} \mathrm{PO}_{4}, 26$ $\mathrm{NaHCO}_{3}, 2.0 \mathrm{MgSO}_{4}, 10$ dextrose, and $2.0 \mathrm{CaCl}_{2}$ continuously bubbled with $95 \% \mathrm{O}_{2}-5 \% \mathrm{CO}_{2}$ (carbogen). Tissue blocks containing the substantia nigra pars compacta ( $\mathrm{SNC}$ ) and its surrounding structures were prepared and sliced at $220-240 \mu \mathrm{m}$ thickness in the tangential plane using a vibrating tissue slicer (Leica VT-1000s; Leica Instruments). After the initial preparation, slices were allowed to equilibrate at either $37^{\circ} \mathrm{C}$ for 30 $\mathrm{min}$ or at room temperature for $1 \mathrm{~h}$, before they were used.

Electrophysiology. Slices were transferred to a recording chamber that was continuously perfused with oxygenated ACSF at a rate of $2-3 \mathrm{ml} /$ $\mathrm{min}$. The temperature was maintained at $35-37^{\circ} \mathrm{C}$. Infrared differential interference contrast video microscopy was used to visualize slices through a $40 \times$ water-immersion lens equipped to either a Zeiss Axioscope (Zeiss) or an Olympus BX51 (Olympus) upright microscope using gradient-contrast illumination. Patch pipettes were pulled from thinwalled borosilicate glass (outer diameter $=1.5 \mathrm{~mm}$, inner diameter $=$ $1.17 \mathrm{~mm}$ ) using either a P-97 or P-87 Flamming-Brown electrode puller (Sutter Instruments). For cell-attached or whole-cell recordings the electrodes were filled with a solution that contained the following (in $\mathrm{mm}$ ): $140.5 \mathrm{KMeSO}_{4}, 0.2$ EGTA, 7.5 NaCl, 10 HEPES, 0.01 phosphocreatine, $2 \mathrm{NaATP}, 0.2 \mathrm{NaGTP}$, adjusted to a $\mathrm{pH}$ of 7.3 with KOH. For this solution we measured a $-7 \mathrm{mV}$ liquid junction potential. The whole-cell solution was also used for perforated-patch recordings, except gramicidin-D (Sigma) was added at a final concentration of 50-100 $\mu \mathrm{g} / \mathrm{ml}$ and sonicated $15-20 \mathrm{~min}$ before recording. Perforated-patch recordings were made in tandem with whole-cell recordings; no clear differences were observed between the two techniques, and thus the data were pooled. While the majority of recordings were obtained with perforated-patch and whole-cell patch-clamp recordings, some neurons were recorded using the minimally invasive, cell-attached configuration. No differences in burst length or frequency were observed in cellattached recordings and they were pooled with the intracellular recordings for frequency and spike count analysis. For whole-cell voltage-clamp recordings pipettes were filled with a solution containing the following (in mM): $135 \mathrm{CsCl}, 5$ QX-314 (Sigma), $2 \mathrm{MgCl}_{2}, 5$ EGTA, 10 HEPES, 0.2 NaGTP, 2 NaATP, adjusted to a $\mathrm{pH}$ of 7.3 with $\mathrm{CsOH}$. The final tip resistance was 5-10 M $\Omega$ for both cell-attached and whole-cell recordings and 2-5 M $\Omega$ for perforated-patch recordings. Recordings were made using either an Axopatch 200B or MultiClamp 700B amplifier (Molecular Devices). Signals were digitized at $10-50 \mathrm{kHz}$ and low pass filtered with a corner frequency of $2-10 \mathrm{kHz}$. Digitized data were saved for offline analysis to a hard disk using PClamp 8.1 software (Molecular Devices) or Axograph X software (Axograph X). Series resistances for whole-cell recordings ranged from 2 to $5 \mathrm{M} \Omega$, and from 10 to $60 \mathrm{M} \Omega$ in perforated-patch recordings. Iontophoresis pipettes (20-80 $\mathrm{M} \Omega$ ) were filled with either $1 \mathrm{M} \mathrm{Na}^{+}$glutamate or $200 \mathrm{~mm}$ NMDA. Typical retaining currents were 0 to $+15 \mathrm{nA}$, and ejection currents were -10 to $-120 \mathrm{nA}$. Dopaminergic neurons were identified by a large $I_{\mathrm{h}}$, long action potential duration $(>1.5 \mathrm{~ms})$, slow spontaneous firing rate $(<10 \mathrm{~Hz})$, and location within SNC (Grace and Bunney, 1983; Kita et al., 1986; Calabresi et al., 1989; Grace and Onn, 1989; Kang and Kitai, 1993). Additional criteria in some neurons included the presence of an inhibitory mGluR response (Marino et al., 2001).

Dynamic clamp. For dynamic clamp experiments we used an $\times 86$ based personal computer running RTXI, a real-time Linux-based data acquisition program (http://rtxi.org), to calculate the current necessary to inject an artificial conductance through a MultiClamp 700B amplifier in current clamp. The dynamic clamp component of this software package is described by Dorval et al. (2001). We could reliably maintain acquisition/update rates of $10 \mathrm{kHz}$. The current flowing through an NMDA receptor $\left(I_{\mathrm{NMDA}}\right)$ was modeled by the following equation: $I_{\mathrm{NMDA}}=\bar{g}_{\mathrm{NMDA}} *\left(V_{\mathrm{m}}-E_{\mathrm{NMDA}}\right) /\left(1+\exp \left[\left(\left[\mathrm{Mg}^{2+}\right]_{\mathrm{o}} / 3.57\right) *\left(-V_{\mathrm{m}} *\right.\right.\right.$ $0.062)])$, where $V_{\mathrm{m}}$ is the membrane voltage in millivolts, $E_{\mathrm{NMDA}}$ is the reversal potential for the NMDA receptor in millivolts, $\left[\mathrm{Mg}^{2+}\right]_{\mathrm{o}}$ is the external $\mathrm{Mg}^{2+}$ millimolar concentration, and $\bar{g}_{\mathrm{NMDA}}$ is the maximal conductance in nanosiemens. The current flowing through an AMPA receptor $\left(I_{\mathrm{AMPA}}\right)$ was modeled by the following equation: $I_{\mathrm{AMPA}}=\bar{g}_{\mathrm{AMPA}}$ $*\left(V_{\mathrm{m}}-E_{\mathrm{AMPA}}\right)$. Many of the glutamatergic inputs to dopaminergic neurons fire at high rates. For example, the subthalamic nucleus can fire at rates beyond $100 \mathrm{~Hz}$ (Isoda and Hikosaka, 2008) and in combination with other excitatory afferents (Geisler et al., 2007), postsynaptic currents rapidly reach a steady-state level (Hestrin et al., 1990). The modeled currents mimic this steady state. All dynamic clamp recordings were done with a balanced bridge with a Multiclamp 700B in continuous current clamp (Bridge Mode).

Chemicals. All chemicals were obtained from Tocris Bioscience or Sigma-Aldrich.

Analysis and statistics. Electrical traces were analyzed off-line using custom-written software or Axograph X and Mathematica 6 or 7 (Wolfram Research). Statistical tests were performed using the software package R (R Foundation for Statistical Computing) or GraphPad Prism (GraphPad Software). A $p$ value of $<0.05$ was used as our significance level for all tests. All numerical data are expressed as the value \pm the sample-corrected SEM. To analyze changes in spike $\mathrm{d} V_{\mathrm{m}} / \mathrm{d} t$, we obtained the peak $\mathrm{d} V_{\mathrm{m}} / \mathrm{d} t$ for each of three spikes: the first in the train, the last in the train, and $100 \mathrm{~ms}$ into the train (or the closest available spike). We obtained difference scores by subtracting each spike's $\mathrm{d} V_{\mathrm{m}} / \mathrm{d} t$ value from that of the first spike in the train, and dividing by the size of the first spike's $\mathrm{d} V_{\mathrm{m}} / \mathrm{d} t$. We then performed two-way repeated-measures ANOVA on these difference scores; the factors were treatment condition and time, i.e., $100 \mathrm{~ms}$ versus last spike. We supplemented this method with Dunnett's multiple-comparison test. Data were tested with twotailed tests and an $\alpha$ value of 0.05 .

\section{Results}

\section{NMDA receptor activation is necessary and sufficient for the generation of burst firing}

We began our studies by determining the influence that both AMPA and NMDA receptors had in the generation of burst firing. We made visualized whole-cell and perforated-patch recordings from putative dopaminergic neurons of the SNC. In the course of our studies we wanted to conduct pharmacological experiments with and without action potentials, so we chose to evoke bursts of action potentials by direct iontophoretic application of glutamate $(50-300 \mathrm{~ms})$ near the recorded neuron at both proximal dendritic and somatic locations. Previous studies demonstrate that such iontophoretic application of glutamate triggers burst firing similar to that evoked by electrical stimulation of glutamatergic afferents (Morikawa et al., 2003).

Iontophoretic application of glutamate triggered transient episodes of high-frequency firing in control ACSF. On average there were $4.9 \pm 1.8$ spikes within episodes in this sample, and they showed an average frequency of $15.9 \pm 6.9 \mathrm{~Hz}$ (Fig. 1A, top). These bursts appear similar to those observed in vivo, in that they show a progressive decrease in spike amplitude and increase in interspike-interval throughout the burst (Grace and Bunney, 1984). Bursts triggered by iontophoretic application to dendritic and somatic locations did not differ from each other (data not shown). Burst firing was not attenuated when 15-25 $\mu \mathrm{M}$ NBQX, an AMPA receptor antagonist, was washed into the bath (Fig. $1 \mathrm{~A}$, middle). In the presence of NBQX the average number of spikes within a burst was $7.3 \pm 4.3$, which was a slight increase in our sample over control conditions $(p<0.01$, one-way ANOVA plus Dunnett's test), but still within the range of bursts observed in vivo (Grace and Bunney, 1984). This small increase in spike number was probably not due to any kind of transient effect of NBQX wash-in, because our sample includes both cells that were acutely and constitutively exposed to NBQX. The average within-burst firing frequency in the presence of NBQX was $17.6 \pm 9.2 \mathrm{~Hz}$, which was not significantly different from those evoked in con- 
trol conditions $(p>0.05$, one-way ANOVA plus Dunnett's test). In contrast, bath application of $50 \mu \mathrm{M} \mathrm{MK}-801$, a selective NMDA receptor antagonist, dramatically reduced the number of spikes associated with the iontophoretic application to $0.9 \pm 1.0(p<0.01$, one-way ANOVA plus Dunnett's test) as well as the average frequency to $4.0 \pm 6.2 \mathrm{~Hz}(p<$ 0.001, one-way ANOVA plus Dunnett's test), relative to control conditions (Fig. $1 A$, bottom). The MK-801 sample contains both cells that were previously exposed to NBQX and those that were not. These cells were pooled because there were no clear differences observed.

Like previous work (Morikawa et al., 2003), we also found that NMDA receptor activation was necessary for triggering burst firing evoked by electrical stimulation. Bursts were also evoked by stimulation of glutamatergic afferents by placing a bipolar stimulating electrode within the SNC. The stimuli consisted of $8-17$ pulses delivered at 50 or $100 \mathrm{~Hz}$. In control conditions, mean burst frequency was $22.5 \pm$ 3.7 Hz $(n=6)$, which was not statistically different from that evoked with iontophoresis ( $p>0.1$; unpaired $t$ test). Bath application of $50 \mu \mathrm{M}$ MK-801 or $20 \mu \mathrm{M} \mathrm{CPP}$ [3-(2-carboxypiperazin-4-yl)propyl-1phosphonic acid] without NBQX abolished burst firing, resulting in a mean firing rate of $1.6 \pm 1.8 \mathrm{~Hz}(n=5 ; p<$ 0.005; unpaired $t$ test) (supplemental Fig. 1, available at www.jneurosci.org as supplemental material). Mean burst frequency, sensitivity to NMDA antagonists, progressive decrease in spike amplitude, and increase in interspike-interval throughout the burst were indistinguishable between bursts induced by electrical stimulation or glutamate iontophoresis, and both of these appear identical to bursts observed in vivo (Grace and Bunney, 1984). Together, these results show that the NMDA glutamate receptor is both a necessary and sufficient element in the generation of highfrequency firing in midbrain dopaminergic neurons.

\section{Activation of glutamate receptors}

\section{and oscillatory inputs counteract depolarization block}

Dopaminergic neurons' spontaneous firing rates are restricted to a narrow range, even when subjected to constant depolarizing current injections. When very large currents are injected into the neuron ( $1 \mathrm{nA}$ or more), they can fire with a short interspike interval, but cannot sustain firing: this phenomenon is known as depolarization block (Grace and Bunney, 1986; Richards et al., 1997). The mechanism of depolarization block is not certain, but it is consistent with the accumulation of $\mathrm{Na}^{+}$channel inactivation. To examine the nature of the depolarization block and whether it could be overcome by an oscillatory current, we compared action potential generation triggered by either injection of a $500 \mathrm{~ms}$, constant 500-900 pA current step, or glutamate iontophoresis (Fig. 2A). This sample of dopaminergic cells fired on average only $3.1 \pm 0.4$ spikes $(n=8)$ in response to a constant current step that was significantly less than what we observed in the same neurons in response to glutamate iontophoresis (average spike count for iontophoresis $=7.1 \pm 0.5, n=57 ; p<0.005$; unpaired $t$ test). Iontophoresis of glutamate onto dopaminergic cells (Fig. 2A, middle trace) elicited trains of 5-10 spikes with mean firing rates between 15 and $20 \mathrm{~Hz}$ and spike amplitude degraded in a more gradual manner than seen during constant current injection. We could reliably trigger high-frequency firing much like that seen with glutamate iontophoresis by applying a high-frequency train of 10-30 ms depolarizing (350-1000 pA) current pulses, interleaved with 5-10 ms hyperpolarizing ( -25 to $-100 \mathrm{pA}$ ) current pulses (the "square-wave" protocol). When we applied the square-wave currents, we were able to elicit spikes on each of the depolarizing pulses (pulse frequency up to $75 \mathrm{~Hz}$ ), demonstrating that an oscillatory current, but not constant depolarization, can support high-frequency firing in dopaminergic neurons.

To determine whether the degree of $\mathrm{Na}^{+}$channel inactivation could underlie the ability of dopaminergic neurons to fire at high frequencies, we differentiated our voltage records, resulting in records of how the temporal derivatives of membrane voltage $\left(\mathrm{d} V_{\mathrm{m}} / \mathrm{d} t\right.$ ) evolve in time (Fig. $\left.2 \mathrm{~B}\right)$. Membrane current is proportional to $\mathrm{d} V_{\mathrm{m}} / \mathrm{d} t$ and the maximum value of $\mathrm{d} V_{\mathrm{m}} / \mathrm{d} t$ occurs during the upstroke of the action potential, when voltage-gated $\mathrm{Na}^{+}$currents dominate, and so the maximum value of $\mathrm{d} V_{\mathrm{m}} / \mathrm{d} t$ is used as a measure of voltage-gated $\mathrm{Na}^{+}$channel availability (Gettes and Reuter, 1974). Spike trains resulting from each protocol showed progressive decreases in the maximum value of $\mathrm{d} V_{\mathrm{m}} / \mathrm{d} t$, consistent with sodium channel inactivation (Fig. $2 B$ ). 

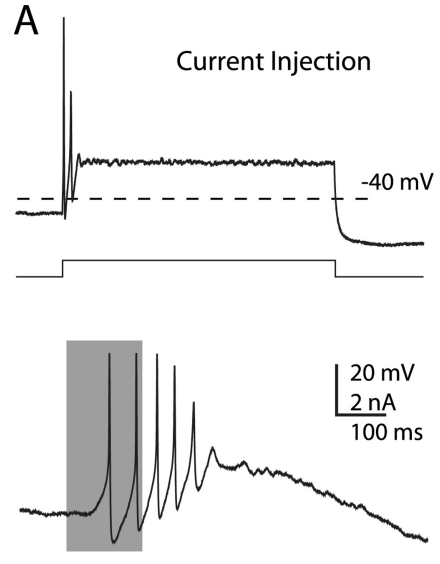

Ionto

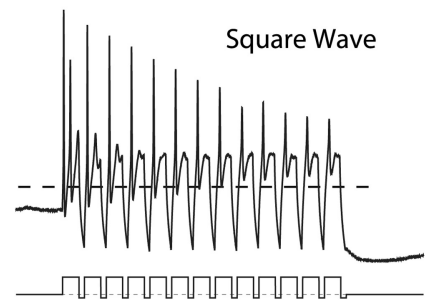

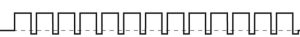

C
B

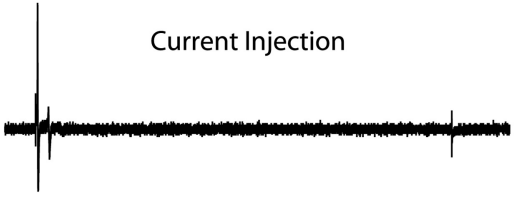

lonto
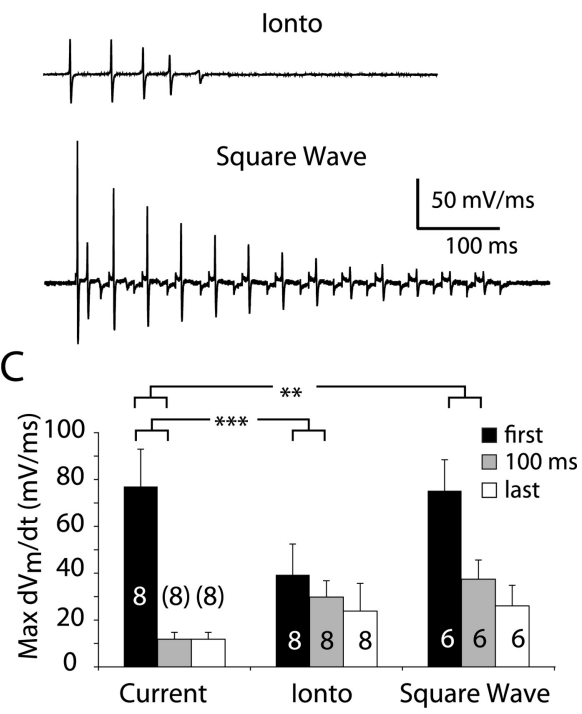

Figure 2. Oscillatory inputs and glutamate iontophoresis counteract depolarization block. $A$, Example traces from the same neuron recorded in the whole-cell configuration. Top, The neuron was injected with a $500 \mathrm{~ms}, 700 \mathrm{pA}$ current injection, to which the neuron rapidly entered a nonspiking depolarized state (Current Injection). Middle, The neuron fired at a rate of $\sim 25 \mathrm{~Hz}$ in response to a 200-ms-duration iontophoretic application of glutamate (Ionto), as indicated by the gray bar. Bottom, The neuron fired repetitively in response to a $25 \mathrm{~Hz}$ train of $35 \mathrm{~ms}, 700 \mathrm{pA}$ current pulses (Square Wave). Note that the pulses are interleaved with 10 ms hyperpolarizing current pulses. $\boldsymbol{B}$, Temporal derivatives $\left(\mathrm{d} V_{\mathrm{m}} / \mathrm{d} t\right)$ of the voltage traces obtained from each protocol in A. C, Maximum d $V_{\mathrm{m}} / \mathrm{d} t$ values, for each protocol, across our sample. Black bars indicate the values for the first spike, whereas gray corresponds to the first spike after a 100 ms latency and white represents the last spike. ${ }^{* *} p<0.01,{ }^{* * *} p<0.001$, two-way ANOVA plus Dunnett's test. The number with each bar is the number of cells in the sample.

Note that the initial maximum value of $\mathrm{d} V_{\mathrm{m}} / \mathrm{d} t$ is much higher when current injections are used as opposed to the iontophoresis; the reason for this is that at the onset of the current injection, the membrane is charged much more rapidly than it would under more physiological conditions. Spikes elicited by constant current injection showed a significantly greater decrease in maximum $\mathrm{d} V_{\mathrm{m}} / \mathrm{d} t$ (spike number $F_{(1,19)}=12.82, p=0.002$; protocol $F_{(2,19)}=$ $10.09, p=0.001$; interaction $\left.F_{(2,19)}=3.48, p=0.0514\right)$ than spikes elicited by either iontophoresis or the square-wave protocol (Fig. $2 C$ ). These results rule out both intrinsic plateau potentials and summation of high-frequency glutamatergic postsynaptic potentials as the mechanism for bursting because both of these would produce prolonged, steady depolarizations, resulting in depolarization block. However, they do not explain why AMPA and NMDA receptor activation should differ.

\section{The voltage sensitivity of the NMDA receptor underlies its ability to trigger burst firing}

To test the possibility that the voltage sensitivity of the NMDA receptor underlies their ability to trigger burst firing, we modified the NMDA receptor's voltage sensitivity by bath application of ACSF that was free of $\mathrm{Mg}^{2+}$ ions. We determined the NMDA receptor's current-voltage relationship ( $I-V$ curve), before and after washing in $\mathrm{Mg}^{2+}$-free ACSF, to determine the degree to which we could manipulate its voltage sensitivity under our conditions. We measured NMDA currents evoked by glutamate iontophoresis, at different voltages, in the presence of 10-15 $\mu \mathrm{M}$ NBQX. Example currents evoked in this way can be seen in Figure
3A. A modified internal solution, containing both $\mathrm{Cs}^{+}$ions and QX-314 (see Materials and Methods), was used to aid in maintaining adequate voltage-control in some of these experiments. The measured $I-V$ curve normally has a negative slope conductance for membrane potential values negative to $-37 \pm 5.6 \mathrm{mV}$, but after 30 min of $\mathrm{Mg}^{2+}$ washout the negative slope conductance region shifts leftwards to begin at $-64 \pm 4.1 \mathrm{mV}$ (Fig. $3 B)(p<0.05$; paired $t$ test). However, even after $>45$ min of $\mathrm{Mg}^{2+}$ washout in every cell tested, the $I-V$ relationship never became linear. There was no change in holding current at $-65 \mathrm{mV}$ during washout of $\mathrm{Mg}^{2+}$ (data not shown). We triggered burst firing by glutamate iontophoresis, while recording membrane voltage in the whole-cell configuration. In control conditions, with 2 $\mathrm{mM} \mathrm{Mg}^{2+}$ in the ACSF, dopaminergic neurons showed an average intraburst frequency of $17.3 \pm 3.3 \mathrm{~Hz}(n=8)$ (Fig. $3 C$, top trace). As the $\mathrm{Mg}^{2+}$-free solution was washed in, dopaminergic neurons began to show an increase in mean intraburst frequency (Fig. 3C, middle trace). On average, after 5-6 min the mean intraburst frequency was $23.8 \pm 5.0 \mathrm{~Hz}(n=8)$, which was not significantly different from control ( $p>0.05$, one-way ANOVA plus Dunnett's test). After 10 min the mean intraburst firing rate was $31.6 \pm 7.1 \mathrm{~Hz}$ $(n=7)$, which was significantly different from control conditions $(p<0.05$, oneway ANOVA plus Dunnett's test). This effect reversed within a few minutes of exposing the slice back to control ACSF (mean intraburst rate $=16.7 \pm 3.3 \mathrm{~Hz} ; n=7)($ Fig. $3 D)$. The number of spikes was not significantly different across any condition (mean number of spikes for control conditions $=4.4 \pm 0.8,5 \mathrm{~min} 0$ $\mathrm{Mg}^{2+}=4.5 \pm 1.0,>10 \min 0 \mathrm{Mg}^{2+}=4.0 \pm 1.1 ; p>0.5$ for all comparisons with control, one-way ANOVA plus Dunnett's test).

These results show that in nominally $\mathrm{Mg}^{2+}$-free solutions, dopaminergic neurons show an increased NMDA-dependent inward current and a hyperpolarized shift in its voltage sensitivity, which is associated with an enhanced ability to fire bursts at higher frequencies, but with the same number of spikes. It is surprising that we were unable to completely remove the voltage sensitivity of the NMDA current in $\mathrm{Mg}^{2+}$-free solution, but these results show the strong dependence of bursting on the strength and voltage dependence of the NMDA conductance. The NMDA receptor's negative slope conductance region does not abruptly shift to hyperpolarized potentials during application of $\mathrm{Mg}^{2+}$ free solution, but gradually moves to hyperpolarized potentials, with the amount of inward current available at hyperpolarized potentials increasing concomitantly (Jahr and Stevens, 1990). Our inability to completely remove the voltage dependence of the NMDA current could be related to failure of our solution to completely eliminate $\mathrm{Mg}^{2+}$ from tissue slices and/or the expression of the NMDA subunit, NR2D, in dopaminergic neurons (Brothwell et al., 2008). Regardless, we would predict that if we could remove the NMDA receptor's voltage sensitivity we would 
disrupt burst firing. We therefore made use of the dynamic clamp technique (Robinson and Kawai, 1993; Sharp et al., 1993; Dorval et al.), which allowed us to impose a modeled NMDA conductance to a live neuron and alter its voltage sensitivity in an unequivocal manner.

We applied a somatic NMDA conductance that was calculated using the description of Jahr and Stevens (1990) (see Materials and Methods), allowing us to vary the effective external $\mathrm{Mg}^{2+}$ concentration $\left(\left[\mathrm{Mg}^{2+}\right]_{\mathrm{o}}\right)$ throughout an experiment. Bursts in dopaminergic neurons are slow, even compared with the activation kinetics of NMDA channels, so we applied constant NMDA conductances, rather than shaping the NMDA conductance to match the activation kinetics of the channel. Within an experiment, we kept the maximal conductance constant and varied $\left[\mathrm{Mg}^{2+}\right]_{\mathrm{o}}$ from 2 to $0 \mathrm{mM}$ in $0.5 \mathrm{~mm}$ decrements. When $\left[\mathrm{Mg}^{2+}\right]_{\mathrm{o}}$ was set to 0 $\mathrm{mm}$ the conductance was linear, and thus behaved like an AMPA-mediated conductance (Fig. 4C). Example traces from a neuron with $\left[\mathrm{Mg}^{2+}\right]_{\mathrm{o}}$ set to 1.5 and 0.0 $\mathrm{mM}$ can be seen in Figure $4 A$. When $\left[\mathrm{Mg}^{2+}\right]_{\mathrm{o}}$ was set between 1 and $2 \mathrm{~mm}$ the neuron fired between 27.0 and $14.8 \mathrm{~Hz}$, which was within the range of burst firing evoked by iontophoresis or electrical stimulation in control media (Fig. 4B). When $\left[\mathrm{Mg}^{2+}\right]_{\mathrm{o}}$ was set to $0.5 \mathrm{~mm}$, the average rate of firing was $54.6 \pm 13.5 \mathrm{~Hz}$, which was similar to what we observed for burst firing evoked in nominally $\mathrm{Mg}^{2+}$ free media. So long as $\left[\mathrm{Mg}^{2+}\right]_{\mathrm{o}}$ was set to a nonzero value, and there was a negative slope conductance in the $I-V$ relation of the applied conductance, neurons remained capable of sustained firing for a few seconds after the conductance onset. We found that the number of spikes occurring within one second of the conductance onset fell within the range of what we observed with iontophoretically evoked bursts (Fig. $4 B$ ). When $\left[\mathrm{Mg}^{2+}\right]_{\mathrm{o}}$ was set to $0 \mathrm{~mm}$, which is a good approximation of a physiological plateau potential, we found that the neurons were capable of discharging only $2.4 \pm 0.2$ spikes, similar to what we observed with constant current injections (compare Fig. $2 A$, top trace, with Fig. $4 A$, bottom trace; $p>0.1$; unpaired $t$ test). The increase in firing rate observed when reducing $\left[\mathrm{Mg}^{2+}\right]_{\mathrm{o}}$ was related to an increase in the amplitude of the negative slope conductance in the voltage range visited by the membrane potential during the interspike interval (Fig. 4C). Even with NMDA conductances, firing often failed after a prolonged period of firing at burst frequencies. We consistently observed that when firing ceased during an NMDA conductance step, there continued to be a prominent membrane potential oscillation (Fig. 4A, top inset). This oscillation was never observed during an AMPA conductance step. We measured the power spectra of the membrane potential in four neurons with $\left[\mathrm{Mg}^{2+}\right]_{\mathrm{o}}$ set to 1.5 and $0 \mathrm{~mm}$. When $\left[\mathrm{Mg}^{2+}\right]_{\mathrm{o}}$ was set to $1.5 \mathrm{~mm}$ we consistently observed a peak in the spectrum close to the frequency of burst firing. No significant peaks were seen when $\left[\mathrm{Mg}^{2+}\right]_{\mathrm{o}}$ was set to $0 \mathrm{mM}$ (Fig. $4 \mathrm{D}$ ). The average peak was centered at $32.4 \pm 4.8 \mathrm{~Hz}$ with the NMDA conductance, and this was not significantly different from the mean firing rate measured at the beginning of the NMDA step, before firing failed (mean firing rate $=20.5 \pm 6.7 \mathrm{~Hz} ; p>0.05$; paired $t$ test).

\section{An action potential-independent, intrinsic, high-frequency oscillation is engaged by NMDA receptor activation} Our results support the idea that the NMDA receptor plays a unique role in driving burst firing because of its voltage dependence. In addition, they provide some evidence that a voltage oscillation accompanies NMDA receptor activation as well. To determine whether these voltage oscillations were action potential independent, we recorded from dopaminergic neurons in current-clamp using either the whole-cell or perforated-patch configuration. When spike generating $\mathrm{Na}^{+}$channels were blocked with TTX $(1 \mu \mathrm{M})$, we consistently observed the ongoing slow voltage oscillation known to drive pacemaking (Fig. $5 A$ ). There were no apparent voltage oscillations in response to applied AMPA conductances, similar to conditions in which spikes were not blocked (Fig. 4A). However, when an NMDA conductance 
A
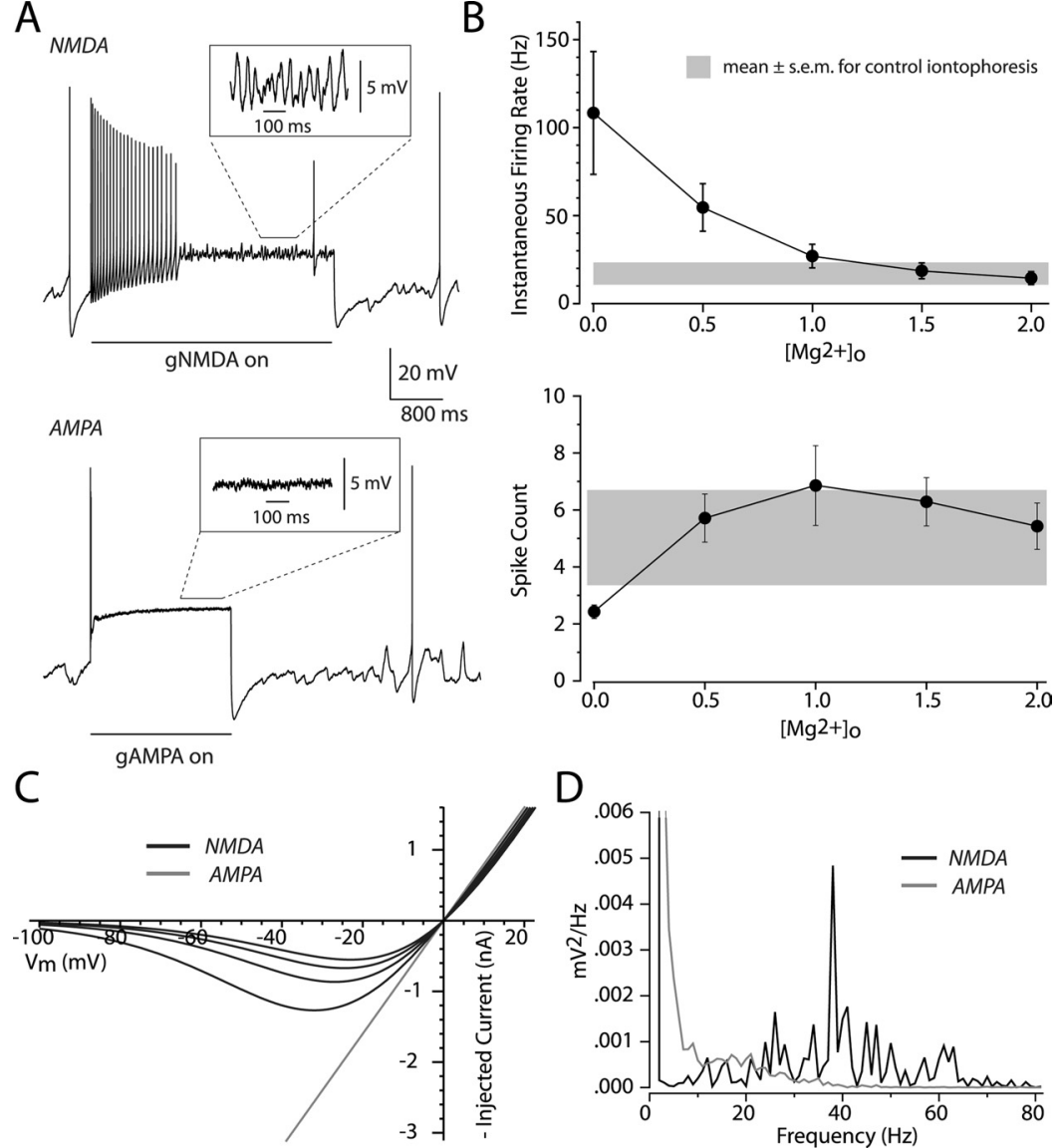

Figure 4. The NMDA receptor's voltage dependence is a critical element in burst firing. $A$, An NMDA or AMPA conductance was applied to the soma of dopaminergic cells recorded in the whole-cell configuration. The conductances were modeled using a formalism for the NMDA receptor by Jahr and Stevens (1990). We treated the external $\mathrm{Mg}^{2+}$ concentration, $\left[\mathrm{Mg}^{2+}\right]_{0}$, as a free parameter and kept the maximal conductance fixed within an experiment. Top, Example trace evoked by turning on a conductance with $\left[\mathrm{Mg}^{2+}\right]_{0}=1.5 \mathrm{~mm}$ (NMDA). Note the presence of prominent oscillations throughout the trace even when spiking has stopped. Bottom, Trace from the same cell with $\left[\mathrm{Mg}^{2+}\right]_{0}=0.0 \mathrm{~mm}$ (AMPA). Note the rapid decrement of spiking and lack of oscillations. $\boldsymbol{B}$, Top, Plot of the average instantaneous firing rate as a function of $\left[\mathrm{Mg}^{2+}\right]_{0}$ for a sample of six cells. The average rate of burst firing ( \pm SEM) evoked with iontophoresis (see Fig. 1) is shown in gray as a reference. Bottom, Plot of the number of spikes counted within $1 \mathrm{~s}$. of the conductance step for the same six cells; again, the distribution of iontophoresis experiments is shown in gray. $C, I-V$ curves for our modeled conductance calculated for different $\left[\mathrm{Mg}^{2+}\right]_{0}$. Black traces are for $\left[\mathrm{Mg}^{2+}\right]_{0}=2.0,1.5,1.0$, and $0.5 \mathrm{~mm}$ (NMDA) from the smallest to largest curves, respectively. Gray trace is for $\left[\mathrm{Mg}^{2+}\right]_{0}=0.0 \mathrm{~mm}$ (AMPA). D, Power spectra after spiking has ceased from the example traces shown in $\boldsymbol{A}$.

was applied to the neuron we consistently observed the same fast voltage oscillations that were observed at the end of spiking when $\mathrm{Na}^{+}$channels were not blocked (Fig. 5A,B). Again, we found that the spike independent voltage oscillations were not significantly different from high-frequency burst firing triggered by NMDA activation (mean control firing rate $=15.4 \pm 1.1 \mathrm{~Hz} ; n=4$; oscillation with peak power $=17.1 \pm 3.5 \mathrm{~Hz} p>0.5$; paired $t$ test).

To determine whether the fast voltage oscillation was due to the interaction of the NMDA receptor with the dopaminergic neuron's intrinsic conductances, we again recorded from dopaminergic neurons in current clamp and blocked action potentials with TTX $(1 \mu \mathrm{M})$ or in some cases lidocaine $(250 \mu \mathrm{M})$. Lidocaine at this concentration abolished spontaneous action potential generation in a reversible manner and gave results similar to TTX, so the data were pooled.

Although we observed voltage oscillations in all neurons exposed to TTX or lidocaine, we included $1 \mathrm{~mm}$ tetraethylammonium in many experiments to facilitate their analysis, which has been shown in previous studies to enhance the amplitude of the slow oscillation with very little impact on its frequency (Nedergaard et al., 1993; Wilson and Callaway, 2000). Glutamate iontophoresis triggered a fast voltage oscillation that had an average frequency of $14.8 \pm 1.2 \mathrm{~Hz}(n=43)$, slightly lower than that of burst firing [control burst firing rate $=21.9 \pm 1.2 \mathrm{~Hz}(n=57) ; p<0.005$, one-way ANOVA plus Dunnett's test] (Fig. $6 A$ ). Most of the depolarizing events within the oscillation exceeded action potential threshold (Fig. 6A), which was determined by finding the maximal value of $\mathrm{d}^{2} V / \mathrm{d} t^{2}$ obtained from voltage recordings before action potentials were blocked. The frequency of these oscillations was reduced by blockade of NMDA receptors, with either $250 \mu \mathrm{M}$ CPP or $50 \mu \mathrm{M}$ MK-801 $(8.3 \pm 1.5 \mathrm{~Hz})$ relative to control oscillations ( $p<0.005$, one-away ANOVA plus Dunnett's test). The frequency of action potential-independent oscillations increased after washing out CPP (Fig. 6A) $(6.2 \pm 0.83$ oscillations at $10.6 \pm 1.2$ oscillations/s). Full-sized action potentials reappeared after washing out lidocaine $(8.2 \pm 0.84$ spikes at $15.0 \pm 3.0 \mathrm{~Hz}$; data not shown). The action potentialindependent oscillations revealed by sodium channel block were also abolished by $20 \mu \mathrm{M}$ isradipine or nimodipine (Fig. $6 \mathrm{~A}$ ), indicating a dependence of the highfrequency oscillations on L-type voltagegated calcium $\left(\mathrm{Ca}^{2+}\right)$ channels. Highfrequency action potential firing, evoked by application of an NMDA conductance step with $\left[\mathrm{Mg}^{2+}\right]_{0}$ set to $1.5 \mathrm{~mm}$, was driven by a fast membrane potential oscillation, which was again revealed by blocking $\mathrm{Na}^{+}$channels with $1 \mu \mathrm{M}$ TTX (Fig. $6 B)$. Subsequent application of isradipine abolished this oscillation (Fig. 6B). We also tested the effects that a lower concentration of nifedipine would have on burst firing. At $10 \mu \mathrm{M}$, nifedipine has been shown to reduce the frequency of spontaneous oscillation by $20-30 \%$ (Mercuri et al., 1994). We found that bursts evoked by NMDA conductance application or iontophoresis of glutamate were attenuated by $\sim 30 \%$ (control iontophoresis mean rate $=15.3 \pm 1.2 \mathrm{~Hz}$; nifedipine iontophoresis mean rate $=10.9 \pm 0.9 \mathrm{~Hz} ; p<0.05$; control dynamic clamp mean rate $=24.1 \pm 0.8 \mathrm{~Hz}$; nifedipine dynamic clamp mean rate $=16.9 \pm 0.5 \mathrm{~Hz} ; p<0.05)$ (supplemental Fig. 2, available at www.jneurosci.org as supplemental material).

The similar sensitivities of the spontaneous slow oscillation and the high-frequency burst oscillation to dihydropyridines suggest that NMDA receptors may enhance the slow spontaneous oscillation that drives pacemaking (Kuznetsov et al., 2006). If this were true, then burst firing should also be sensitive to blockade of the outward current, which provides the repolarization needed to maintain the oscillation. The outward current most critical for the slow spontaneous oscillation is mediated by the SK channel (Nedergaard et al., 1993; Ping and Shepard, 1996; Amini et al., 1999). We evoked bursts by iontophoresis of glutamate before 
and after blockade of SK channels by 100 nM apamin, which is a selective blocker of SK channels (Ishii et al., 1997). In control conditions the average frequency of burst firing was $18.5 \pm 2.6 \mathrm{~Hz}$. Apamin caused a significant increase in burst frequency, which was $26.6 \pm 2.0 \mathrm{~Hz}(p<0.05 ; n=6$; paired $t$ test), but no significant increase in the number of spikes (control spike count $=4.6 \pm 0.4$, apamin spike count $=$ $6.4 \pm 0.8 ; p>0.5$; paired $t$ test) (Fig. $7 A, B)$. Thus, while both spontaneous and burst firing depend on L-type $\mathrm{Ca}^{2+}$ channel activation, SK channels are not a critical component for burst firing.

\section{Discussion}

Our results demonstrate that the mechanism for glutamate-induced bursting consists of a high-frequency, action potential-independent oscillation that is dependent on NMDA receptor activation and subsequent interaction with intrinsic conductances. Somatic and dendritic NMDA receptor activation is equally capable of triggering burst firing, so the mechanism that underlies the engagement of the fast oscillation does not depend on surface area-to-volume differences. NMDA receptors are uniquely capable of engaging a fast voltage oscillation, which drives burst firing, because of its voltage dependence. During each cycle of the oscillation, the NMDA receptor undergoes $\mathrm{Mg}^{2+}$ block to enhance the hyperpolarizing phase of the oscillation and mitigate depolarization block, and then unblocks during the depolarizing phase, increasing the amplitude of the bursting oscillation. Therefore, the voltage dependence of NMDA receptors allows dopaminergic neurons to fire at rates higher than those possible during sustained depolarization.

\section{The mechanism of burst firing in} midbrain dopaminergic neurons Kuznetsov et al. (2006) proposed a model for burst firing based on evidence that the dopaminergic neuron behaves like a system of coupled oscillators (Wilson and Callaway, 2000). In this model, the L-type $\mathrm{Ca}^{2+}$ and SK currents that underlie the membrane potential oscillation that drives spontaneous firing are distributed equally among the soma and dendrites. Because the surface area-to-volume ratio of the dendrite is much larger than that of the soma, the natural frequency of the dendrite is expected to be much higher. During spontaneous oscillations, the soma generates larger currents and so normally dominates the frequency of the voltagecoupled system. Kuznetsov et al. (2006)
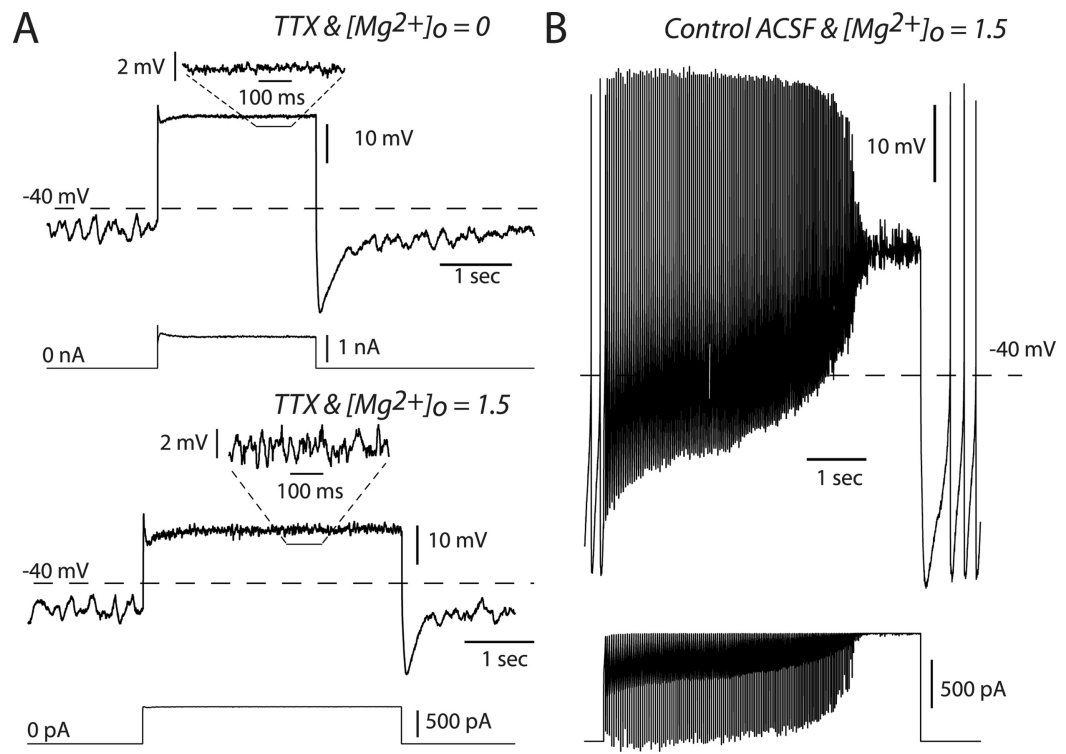

Figure 5. NMDA, but not AMPA, conductances engage a high-frequency, action potential-independent oscillation. $\boldsymbol{A}$, Dynamic clamp experiments performed in the presence of TTX and recorded in the whole-cell configuration. Top, Example electrical trace obtained for $\left[\mathrm{Mg}^{2+}\right]_{0}=0.0 \mathrm{~mm}$ with the injected current below. Just before the conductance step, a low-frequency oscillation can be seen. During the conductance step the membrane voltage remains flat. Bottom, Same as top except $\left[\mathrm{Mg}^{2+}\right]_{0}=1.5 \mathrm{~mm}$. During the conductance step a higher-frequency oscillation (relative to that before the step) is observed. Below each voltage trace is the injected current waveform. $\boldsymbol{B}$, Control firing evoked by applying the same NMDA conductance step $\left(\left[\mathrm{Mg}^{2+}\right]_{0}=1.5\right)$ as in $\boldsymbol{A}$, before applying TTX.

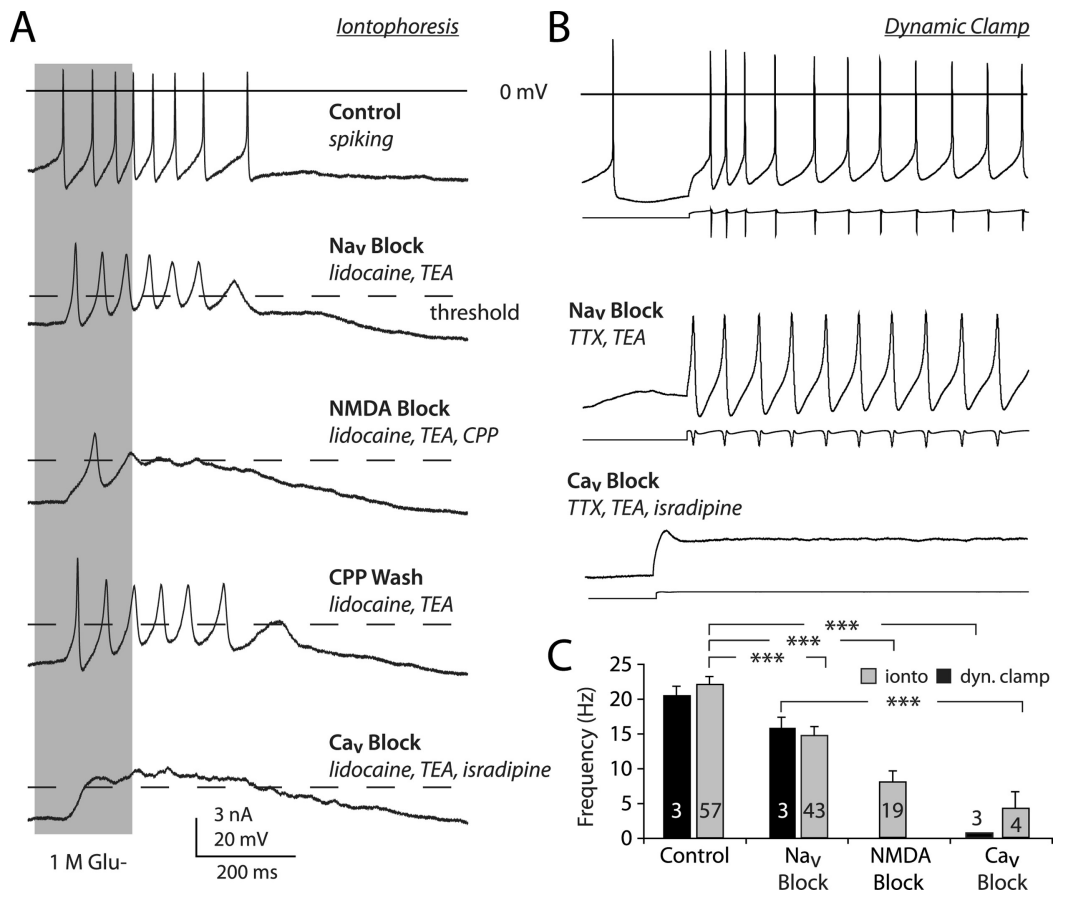

Figure 6. NMDA receptor activation triggers an intrinsic, action potential-independent, voltage oscillation. $\boldsymbol{A}$, lontophoresis of glutamate (gray bar) elicited a burst of action potentials from a cell recorded in the whole-cell configuration. Blockade of sodium channels ( $\mathrm{Na}_{\mathrm{v}}$ block) revealed large voltage oscillations that were attenuated by NMDA and calcium channel antagonists (NMDA and $C \mathrm{a}_{\mathrm{v}}$ block, respectively). Note that all examples are from the same cell with experiments done sequentially. Dashed line indicates spike threshold. $\boldsymbol{B}$, Same experiment as in $\boldsymbol{A}$, except dynamic clamp was used to mimic NMDA receptor conductance. Below each voltage trace is the waveform of the injected current. $\boldsymbol{C}$, Bar chart showing the frequency of glutamate-elicited oscillations before and after $\mathrm{Na}_{\mathrm{v}}$ block, NMDA block, or $\mathrm{Ca}_{\mathrm{v}}$ block in iontophoresis (ionto) experiments (gray) and dynamic clamp (dyn. clamp) experiments (black). The number with each bar is the number of cells in the sample. ${ }^{* *} p<0.001$, Kruskal-Wallis test (score $=42.01, p<0.0001$ ) plus Dunnett's test. TEA, Tetraethylammonium. 
A
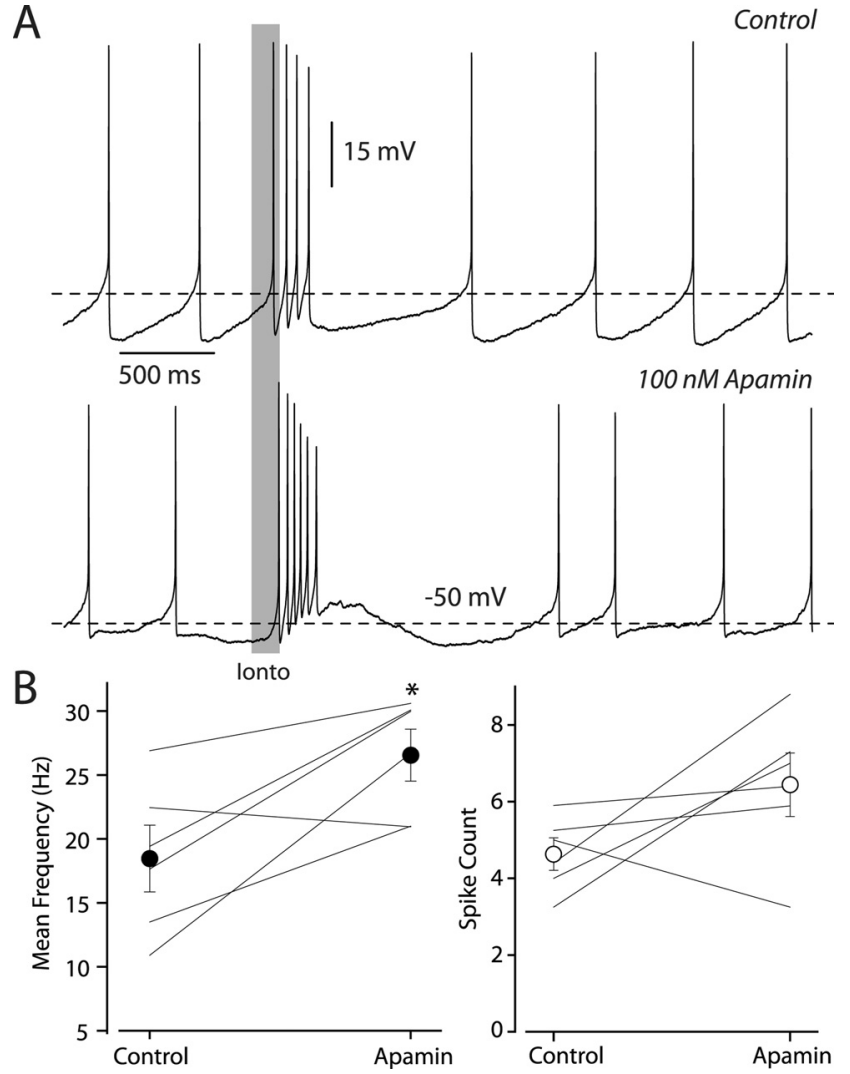

Figure 7. Burst firing is augmented, not suppressed, by SK channel blockade. $\boldsymbol{A}$, Example traces from the same neuron recorded in the whole-cell configuration. Top, Spiking elicited in control media with glutamate iontophoresis (lonto), indicated by gray bar. Bottom, Under the same conditions except with the addition of $100 \mathrm{~nm}$ apamin. Now the cell responds with a much higher frequency and more spikes. $\boldsymbol{B}$, Summary data of the effect of $100 \mathrm{~nm}$ apamin, on both the mean burst firing rate (left) and the total number of spikes within the burst (right), in our sample. Each cell's values before and after drug application are indicated by individual joined lines, and the mean and SEM of our sample are given by the closed and filled circles. Asterisk indicates statistical significance ( $p<0.05$; paired $t$ test).

proposed that the feature that distinguished NMDA receptor activation from that of AMPA receptors, was that NMDA receptors can "act as a current amplifier," because of its voltage dependence and rapid kinetics. Because of this property, NMDA receptor activation was thought to amplify the normally diminutive, but faster, dendritic oscillation without altering its natural frequency.

Our results indicate that there is no clear difference in mechanism between somatic and dendritically localized inputs in generating bursts. We routinely evoked bursts using both somatic and dendritic iontophoresis. We were also able to drive bursts by application of a point NMDA conductance in the soma by conductance clamp. These findings are in contradiction to the model proposed by Kuznetsov et al. (2006), which predicts that NMDA conductance in the soma would reinforce the slow spontaneous oscillation, not the fast one responsible for bursts. However, our results also show the oscillation that drives burst firing is not the same as the oscillation that drives pacemaking. Although burst firing and the underlying oscillation are sensitive to blockade of voltage-gated $\mathrm{Ca}^{2+}$ channels, it is actually enhanced by SK channel blockade. If the oscillation that drives pacemaking were enhanced by NMDA activation we would have predicted that SK channel blockade would abolish burst firing. Therefore, NMDA receptor activation appears to engage a different set of ionic currents, which results in the expression of an otherwise subliminal fast oscillation. While the specific kinetics of the binding and unbinding of $\mathrm{Mg}^{2+}$ could play a role in determining burst frequency, we did not alter the rapid (submillisecond) kinetics assumed in our dynamic clamp experiments. Nevertheless, we found that the burst frequencies obtained with dynamic clamp and iontophoresis were similar. This suggests that the kinetics of the NMDA-recruited fast oscillation are determined by the ion channels that comprise it.

Our results are consistent with a primary role for the L-type $\mathrm{Ca}^{2+}$ channel in providing the depolarizing current for the fast oscillation, as it does for the slow spontaneous oscillation (Nedergaard et al., 1993; Mercuri et al., 1994). However, the hyperpolarizing phase of the burst oscillation cannot arise primarily from SK current. Our results suggest that some other outward current plays this role during bursts. The identity of this outward current is not known, but it must be activated by subthreshold voltages, and is therefore likely to be a voltage-sensitive rather than a calcium-dependent $\mathrm{K}^{+}$current, or delayed rectifier. Dopaminergic neurons are known to possess the subthreshold activated A-type $\mathrm{K}^{+}$current, mediated by the $\mathrm{K}_{\mathrm{v}} 4.3$ subunit, throughout its somatodendritic extent (Liss et al., 2001; Gentet and Williams, 2007). This current, which has kinetics consistent with the fast oscillation, appears to be a powerful modulator of dopaminergic neuron pacemaking (Segev and Korngreen, 2007; Khaliq and Bean, 2008; Putzier et al., 2009), but may also play a central role in burst firing. The use of an L-type $\mathrm{Ca}^{2+}$ current for the depolarizing phases of the oscillation may just be a consequence of its availability, but because a $\mathrm{Ca}^{2+}$-sensitive $\mathrm{K}^{+}$channel is not needed to repolarize the oscillation, it is not obligatory. Dopaminergic neurons are capable of finding new combinations of currents to maintain their pacemaking regardless of the voltage-dependent $\mathrm{Ca}^{2+}$ current availability (Chan et al., 2007; Guzman et al., 2009). While it is not known whether under conditions of $\mathrm{Ca}^{2+}$ channel downregulation dopaminergic neurons will still burst, our results do not rule this out.

\section{The synaptic origin of dopaminergic neuron burst firing}

Stimulation of glutamatergic inputs onto dopaminergic neurons activates both NMDA and AMPA receptors, as at other glutamatergic synapses throughout the CNS (Mereu et al., 1991). What then is the role of AMPA receptors in the generation of burst firing or shaping the output of dopaminergic neurons? Our results show burst firing can occur in the absence of AMPA receptors and argue that NMDA receptors are critical; they do not argue that AMPA receptors cannot be involved at all. Both iontophoresis and electrical stimulation are capable of evoking bursts (Morikawa et al., 2003; Blythe et al., 2007). Blythe et al. (2007) found that bath application of either AMPA or NMDA receptor antagonists attenuated burst firing evoked by highfrequency trains of electrical stimulation, arguing that NMDA and AMPA receptors worked in concert to generate burst firing. This is seemingly contradictory to the present results and previous in vivo results (Overton and Clark, 1992; Chergui et al., 1993; Tong et al., 1996a) showing that NMDA receptor activation is sufficient and necessary in generating bursts. One possibility is that some amount of sustained depolarization may be beneficial for bursting by depolarizing the cell into the membrane potential range of the negative slope conductance region of the NMDA receptor. Below that voltage range, NMDA channels alone are ineffective because of $\mathrm{Mg}^{2+}$ block. It is also possible that periodic synchronous activation of AMPA receptors by rhythmic stimulation of afferents could produce an extrinsic oscillatory input that could drive bursting in a way similar to the square-wave 
current injections shown in Figure 2. As AMPA current is increased, however, it will work against bursting by reducing the hyperpolarizing part of the interspike interval, and promoting depolarization block. This explains why specific disruption of NMDA receptors on dopaminergic neurons can selectively prevent bursting in animals with effectively normal AMPA receptormediated synaptic transmission (Zweifel et al., 2008, 2009).

Burst firing of dopaminergic neurons is a behaviorally important signal necessary for normal reward learning (Schultz, 1997; Tsai et al., 2009; Zweifel et al., 2009). Dopaminergic neurons fire bursts in response to unexpected rewards, or to stimuli that have become predictors of reward, and for conditioned stimuli the intraburst firing rate scales according to the probability of reward associated with that stimulus (Morris et al., 2004). Burst firing in response to a reward is suppressed if the reward is preceded by a stimulus that reliably predicts the reward. A key issue for the mechanism of reward prediction is whether the conditioning mechanism, which assigns the burst to a conditioned stimulus on the basis of its predictive value, is located in the substantia nigra or in other brain regions and is only relayed by the dopaminergic neuron. Our results suggest that the input triggering bursts is a glutamatergic input that acts on NMDA receptors, and that the intraburst frequency is determined by the degree of NMDA receptor activation and the kinetics of the channels underlying the burst oscillation.

Consistent with this view are the findings of Harnett et al. (2009), which find upregulation of NMDA receptor activity with a concomitant reduction in AMPA EPSCs, using an induction protocol that mimicked cue-reward association. Both of these effects would act to enhance synaptically triggered burst firing, which may underlie the acquisition of the burst response to a reward-predicting stimulus (Harnett et al., 2009).

Our results provide a cellular mechanism for the NMDAspecific nature of bursting in dopaminergic neurons. They also suggest that many of the features of dopaminergic cell bursting, including intraburst firing rate and burst duration, arise largely from the kinetics of ion channels on the dopaminergic cells and may be controlled by modulation acting locally within the substantia nigra (Paladini et al., 2001). Our results show that intrinsic cellular dynamics are an important part of synaptic integration in the dopaminergic neuron and should provide a framework for understanding how the dopaminergic neuron's intrinsic properties ultimately influence behavior.

\section{References}

Amini B, Clark JW Jr, Canavier CC (1999) Calcium dynamics underlying pacemaker-like and burst firing oscillations in midbrain dopaminergic neurons: a computational study. J Neurophysiol 82:2249-2261.

Blythe SN, Atherton JF, Bevan MD (2007) Synaptic activation of dendritic AMPA and NMDA receptors generates transient high-frequency firing in substantia nigra dopamine neurons in vitro. J Neurophysiol 97:2837-2850.

Brothwell SL, Barber JL, Monaghan DT, Jane DE, Gibb AJ, Jones S (2008) NR2B- and NR2D-containing synaptic NMDA receptors in developing rat substantia nigra pars compacta dopaminergic neurones. J Physiol 586:739-750.

Calabresi P, Lacey MG, North RA (1989) Nicotinic excitation of rat ventral tegmental neurones in vitro studied by intracellular recording. Br J Pharmacol 98:135-140.

Canavier CC (1999) Sodium dynamics underlying burst firing and putative mechanisms for the regulation of the firing pattern in midbrain dopamine neurons: a computational approach. J Comput Neurosci 6:49-69.

Chan CS, Guzman JN, Ilijic E, Mercer JN, Rick C, Tkatch T, Meredith GE, Surmeier DJ (2007) 'Rejuvenation' protects neurons in mouse models of Parkinson's disease. Nature 447:1081-1086.

Chergui K, Charléty PJ, Akaoka H, Saunier CF, Brunet JL, Buda M, Svensson
TH, Chouvet G (1993) Tonic activation of NMDA receptors causes spontaneous burst discharge of rat midbrain dopamine neurons in vivo. Eur J Neurosci 5:137-144.

Dorval AD, Christini DJ, White JA (2001) Real-Time linux dynamic clamp: a fast and flexible way to construct virtual ion channels in living cells. Ann Biomed Eng 29:897-907.

Geisler S, Derst C, Veh RW, Zahm DS (2007) Glutamatergic afferents of the ventral tegmental area in the rat. J Neurosci 27:5730-5743.

Gentet LJ, Williams SR (2007) Dopamine gates action potential backpropagation in midbrain dopaminergic neurons. J Neurosci 27:1892-1901.

Gettes LS, Reuter H (1974) Slow recovery from inactivation of inward currents in mammalian myocardial fibres. J Physiol 240:703-724.

Grace AA, Bunney BS (1983) Intracellular and extracellular electrophysiology of nigral dopaminergic neurons-1. Identification and characterization. Neuroscience 10:301-315.

Grace AA, Bunney BS (1984) The control of firing pattern in nigral dopamine neurons: burst firing. J Neurosci 4:2877-2890.

Grace AA, Bunney BS (1986) Induction of depolarization block in midbrain dopamine neurons by repeated administration of haloperidol: analysis using in vivo intracellular recording. J Pharmacol Exp Ther 238:10921100.

Grace AA, Onn SP (1989) Morphology and electrophysiological properties of immunocytochemically identified rat dopamine neurons recorded in vitro. J Neurosci 9:3463-3481.

Guzman JN, Sánchez-Padilla J, Chan CS, Surmeier DJ (2009) Robust pacemaking in substantia nigra dopaminergic neurons. J Neurosci 29:11011-11019.

Harnett MT, Bernier BE, Ahn KC, Morikawa H (2009) Burst-timingdependent plasticity of NMDA receptor-mediated transmission in midbrain dopamine neurons. Neuron 62:826-838.

Hestrin S, Sah P, Nicoll RA (1990) Mechanisms generating the time course of dual component excitatory synaptic currents recorded in hippocampal slices. Neuron 5:247-253.

Hollerman JR, Schultz W (1998) Dopamine neurons report an error in the temporal prediction of reward during learning. Nat Neurosci 1:304-309.

Hyland BI, Reynolds JN, Hay J, Perk CG, Miller R (2002) Firing modes of midbrain dopamine cells in the freely moving rat. Neuroscience 114:475-492.

Ishii TM, Maylie J, Adelman JP (1997) Determinants of apamin and d-tubocurarine block in SK potassium channels. J Biol Chem 272:23195-23200.

Isoda M, Hikosaka O (2008) Role for subthalamic nucleus neurons in switching from automatic to controlled eye movement. J Neurosci 28:7209-7218.

Jahr CE, Stevens CF (1990) Voltage dependence of NMDA-activated macroscopic conductances predicted by single-channel kinetics. J Neurosci 10:3178-3182.

Ji H, Shepard PD (2006) SK Ca2+-activated K+ channel ligands alter the firing pattern of dopamine-containing neurons in vivo. Neuroscience 140:623-633.

Johnson SW, Seutin V, North RA (1992) Burst firing in dopamine neurons induced by N-methyl-D-aspartate: role of electrogenic sodium pump. Science 258:665-667.

Kang Y, Kitai ST (1993) A whole cell patch-clamp study on the pacemaker potential in dopaminergic neurons of rat substantia nigra compacta. Neurosci Res 18:209-221.

Khaliq ZM, Bean BP (2008) Dynamic, nonlinear feedback regulation of slow pacemaking by A-type potassium current in ventral tegmental area neurons. J Neurosci 28:10905-10917.

Kita T, Kita H, Kitai ST (1986) Electrical membrane properties of rat substantia nigra compacta neurons in an in vitro slice preparation. Brain Res 372:21-30.

Kuznetsov AS, Kopell NJ, Wilson CJ (2006) Transient high-frequency firing in a coupled-oscillator model of the mesencephalic dopaminergic neuron. J Neurophysiol 95:932-947.

Li YX, Bertram R, Rinzel J (1996) Modeling N-methyl-D-aspartate-induced bursting in dopamine neurons. Neuroscience 71:397-410.

Liss B, Franz O, Sewing S, Bruns R, Neuhoff H, Roeper J (2001) Tuning pacemaker frequency of individual dopaminergic neurons by Kv4.3L and KChip3.1 transcription. EMBO J 20:5715-5724.

Marino MJ, Wittmann M, Bradley SR, Hubert GW, Smith Y, Conn PJ (2001) Activation of group I metabotropic glutamate receptors produces a direct 
excitation and disinhibition of GABAergic projection neurons in the substantia nigra pars reticulata. J Neurosci 21:7001-7012.

Mayer ML, Westbrook GL, Guthrie PB (1984) Voltage-dependent block by Mg2+ of NMDA responses in spinal cord neurones. Nature 309:261-263.

Mercuri NB, Bonci A, Calabresi P, Stratta F, Stefani A, Bernardi G (1994) Effects of dihydropyridine calcium antagonists on rat midbrain dopaminergic neurones. Br J Pharmacol 113:831-838.

Mereu G, Costa E, Armstrong DM, Vicini S (1991) Glutamate receptor subtypes mediate excitatory synaptic currents of dopamine neurons in midbrain slices. J Neurosci 11:1359-1366.

Morikawa H, Khodakhah K, Williams JT (2003) Two intracellular pathways mediate metabotropic glutamate receptor-induced $\mathrm{Ca}^{2+}$ mobilization in dopamine neurons. J Neurosci 23:149-157.

Morris G, Arkadir D, Nevet A, Vaadia E, Bergman H (2004) Coincident but distinct messages of midbrain dopamine and striatal tonically active neurons. Neuron 43:133-143.

Nedergaard S, Flatman JA, Engberg I (1993) Nifedipine- and omegaconotoxin-sensitive $\mathrm{Ca} 2+$ conductances in guinea-pig substantia nigra pars compacta neurones. J Physiol 466:727-747.

Nowak L, Bregestovski P, Ascher P, Herbet A, Prochiantz A (1984) Magnesium gates glutamate-activated channels in mouse central neurones. Nature 307:462-465.

Overton P, Clark D (1992) Iontophoretically administered drugs acting at the $\mathrm{N}$-methyl-D-aspartate receptor modulate burst firing in A9 dopamine neurons in the rat. Synapse 10:131-140.

Paladini CA, Fiorillo CD, Morikawa H, Williams JT (2001) Amphetamine selectively blocks inhibitory glutamate transmission in dopamine neurons. Nat Neurosci 4:275-281.

Ping HX, Shepard PD (1996) Apamin-sensitive Ca(2+)-activated K+ channels regulate pacemaker activity in nigral dopamine neurons. Neuroreport 7:809-814.

Putzier I, Kullmann PH, Horn JP, Levitan ES (2009) Dopamine neuron responses depend exponentially on pacemaker interval. J Neurophysiol 101:926-933.

Richards CD, Shiroyama T, Kitai ST (1997) Electrophysiological and immunocytochemical characterization of GABA and dopamine neurons in the substantia nigra of the rat. Neuroscience 80:545-557.
Robinson HP, Kawai N (1993) Injection of digitally synthesized synaptic conductance transients to measure the integrative properties of neurons. J Neurosci Methods 49:157-165.

Schultz W (1986) Responses of midbrain dopamine neurons to behavioral trigger stimuli in the monkey. J Neurophysiol 56:1439-1461.

Schultz W (1997) Dopamine neurons and their role in reward mechanisms. Curr Opin Neurobiol 7:191-197.

Segev D, Korngreen A (2007) Kinetics of two voltage-gated K+ conductances in substantia nigra dopaminergic neurons. Brain Res 1173:27-35.

Seutin V, Johnson SW, North RA (1993) Apamin increases NMDA-induced burstfiring of rat mesencephalic dopamine neurons. Brain Res 630:341-344.

Sharp AA, O’Neil MB, Abbott LF, Marder E (1993) Dynamic clamp: computer-generated conductances in real neurons. J Neurophysiol 69:992-995.

Tong ZY, Overton PG, Clark D (1996a) Antagonism of NMDA receptors but not AMPA/kainate receptors blocks bursting in dopaminergic neurons induced by electrical stimulation of the prefrontal cortex. J Neural Transm 103:889-904.

Tong ZY, Overton PG, Clark D (1996b) Stimulation of the prefrontal cortex in the rat induces patterns of activity in midbrain dopaminergic neurons which resemble natural burst events. Synapse 22:195-208.

Tsai HC, Zhang F, Adamantidis A, Stuber GD, Bonci A, de Lecea L, Deisseroth K (2009) Phasic firing in dopaminergic neurons is sufficient for behavioral conditioning. Science 324:1080-1084.

Wilson CJ, Callaway JC (2000) Coupled oscillator model of the dopaminergic neuron of the substantia nigra. J Neurophysiol 83:3084-3100.

Yung WH, Häusser MA, Jack JJ (1991) Electrophysiology of dopaminergic and non-dopaminergic neurones of the guinea-pig substantia nigra pars compacta in vitro. J Physiol 436:643-667.

Zweifel LS, Argilli E, Bonci A, Palmiter RD (2008) Role of NMDA receptors in dopamine neurons for plasticity and addictive behaviors. Neuron 59:486-496.

Zweifel LS, Parker JG, Lobb CJ, Rainwater A, Wall VZ, Fadok JP, Darvas M, Kim MJ, Mizumori SJ, Paladini CA, Phillips PE, Palmiter RD (2009) Disruption of NMDAR-dependent burst firing by dopamine neurons provides selective assessment of phasic dopamine-dependent behavior. Proc Natl Acad Sci U S A 106:7281-7288. 